\title{
A Cross-Ethnic Study of Adolescents' Depressed Mood and the Erosion of Parental and Peer Warmth During the Transition to Young Adulthood
}

\author{
William Y. Chung, Chuansheng Chen, Ellen Greenberger, and \\ Jutta Heckhausen
}

University of California, Irvine

This 1-year longitudinal study investigated the effects of adolescents' depressed mood on perceived parental and peer warmth during the transition to young adulthood. We hypothesized that ethnicity would moderate such effects. As part of a larger study, 511 adolescents (154 European, 205 Hispanic, and 152 Asian Americans) participated in this research. They were surveyed shortly before their high school graduation and again 1 year later. Analyses based on 2-wave cross-lagged models showed that higher levels of initial depressive symptoms predicted lower levels of subsequent perceived parental and peer warmth for European Americans. For Asian Americans, higher initial depressed mood was significantly associated with lower levels of perceived peer warmth and was marginally associated with lower levels of parental warmth. In contrast, the erosion of parental and peer warmth was not evident for Hispanic Americans. The role of culture in the erosion of parental and peer warmth during life transitions was discussed.

Accompanied by rapid physical, psychological, and social changes, adolescence represents a particularly vulnerable developmental phase. Depressive symptoms are one of the most common psychological difficulties during adolescence. For example, in a recent study of young adolescents (12 and 13 years old), Kubik, Lytle, Birnbaum, Murray, and Perry (2003) reported a prevalence rate of $35 \%$ for elevated depressive symptoms (indicated by scores of 16 or higher on the 20-item Center for Epidemiologic Studies Depression Scale [CES-D; Radloff, 1977]). The prevalence rate was higher for Asian Americans (47\%) and Hispanic Americans (40\%) than for European Americans (30\%) (Kubik et al., 2003).

Requests for reprints should be sent to Chuansheng Chen, Department of Psychology and Social Behavior, 3340 Social Ecology II, University of California, Irvine, CA 92697-7085. E-mail: cschen@uci.edu 
Researchers have long been interested in the impact of parents and peers on adolescents' depressed mood. Perceived support or warmth from parents and peers has been most consistently linked to amelioration of adolescents' depressed mood based on both cross-sectional (e.g., Greenberger \& Chen, 1996; Greenberger, Chen, Tally, \& Dong, 2000; Parker \& Asher, 1993) and longitudinal data (e.g., Brendgen, Wanner, Morin, \& Vitaro, 2005; Colarossi \& Eccles, 2000; Galambos, Barker, \& Krahn, 2006; Ge, Best, Conger, \& Simons, 1996). These studies are consistent with the hypothesis that supportive relationships with parents and peers reduce depressive symptoms.

Much less is known about the inverse causal direction, that is, whether adolescents' depressed mood leads to an erosion of social support (e.g., Stice, Ragan, \& Randall, 2004). According to the support erosion hypothesis, individuals with depressed mood may erode their relations with others by means of negative self-appraisals, pessimistic outlook, complaints, and reassurance seeking. Evidence from experimental studies has provided some support for this hypothesis. In these experiments, participants were asked to interact briefly (e.g., in dyadic face-to-face interactions or telephone conversations) with depressed targets, nondepressed psychiatric patients, and normal controls. After the interactions, participants reported that they were less willing to engage in future interactions with depressed individuals than with individuals in the other two groups (e.g., Coyne, 1976; Hammen \& Peters, 1977; Joiner, 1999; see King \& Heller, 1984, for an exception). Although these studies are helpful in testing the support erosion hypothesis, participants interacted with strangers with whom they did not have a prior history of either providing or receiving social support (thus no support to be eroded). Longitudinal research focusing on relationships in one's social network is needed to better examine the support erosion hypothesis.

So far, only a few studies, using longitudinal data from samples of predominantly White participants, have examined the possibility of erosion of emotional support for adolescents with depressive symptoms (Compas, Wagner, Slavin, \& Vannatta, 1986; Prinstein, Borelli, Cheah, Simon, \& Aikins, 2005; Sheeber, Hops, Alpert, Davis, \& Andrews, 1997; Slavin \& Rainer, 1990; Stice et al., 2004; Young, Berenson, Cohen, \& Garcia, 2005). Results of these studies were mixed. Two of these studies (Sheeber et al., 1997; Young et al., 2005) found no evidence for emotional support erosion (i.e., depressive symptoms were not associated with subsequent changes in emotional support or relationship quality). The other four found some evidence for the erosion of emotional support hypothesis. Compas et al. (1986) found that late adolescents' depressed mood had a marginally significant correlation with subsequent decreases in social support. In that study, the specific source of social support was not examined.

In a study that differentiated family emotional support from peer emotional support, Slavin and Rainer (1990) found that, for female high school students, initial depressive symptoms were associated with a subsequent 
decrease in family emotional support (from three family members nominated by the adolescents as providing the most support), but not in peer support. In a recent study of early adolescent females, however, Stice et al. (2004) contrasted peer support directly with parental emotional support (instead of overall family support). They found that initial depressive symptoms and major depression predicted future decreases in peer emotional support but not parental support. Consistent with the Stice et al. findings, Prinstein et al. (2005) found that initial levels of early adolescents' depressive symptoms predicted deteriorating friendship quality and stability among girls. Parental factors were not included in that study.

Stice et al. (2004) argued that this pattern of results makes sense because peers have more leeway than parents to withdraw their emotional support from adolescents with increasingly depressed mood. Parents are socially sanctioned to maintain a high level of support for their adolescents even in the face of adversity. Among the many differences between the studies of Slavin and Rainer and Stice et al., age of the research participants and the definition of family support may account for the discrepancies in their results. That is, Stice et al. found no emotional support erosion with a younger sample and a measure of parental support, whereas Slavin and Rainer found erosion of emotional support with an older sample and a measure of family emotional support. It is possible that the social sanctions for consistent support are stronger for parents, especially when their children are young, than for other family members such as siblings or extended family members.

The objective of this study was to extend previous research, especially that of Stice et al. (2004) and Slavin and Rainer (1990), by examining the relations between depressed mood and warmth during the initial period of transition to adulthood and by investigating possible ethnic differences in the extent to which each of these variables impacts the other. In most of the previous studies that examined the social support erosion hypothesis, support was defined as emotional in nature. For example, Stice et al. (2004) specifically focused on the decline in the emotional components of social support, including companionship, guidance, intimacy, affection, admiration, and reliable alliance. Similarly, Slavin and Rainer (1990) and Young et al. (2005) also focused on emotional support. To be consistent with this conceptualization of social support and given the similarities between emotional support and warmth, we have opted to examine emotional support in the form of parental and peer warmth. Parental and peer warmth refers to the acceptance, love, affection, support, care, comfort, and nurturance that parents and peers provide (Rohner, 2004).

Social expectations regarding the appropriate level of parental warmth or emotional support are not uniform across developmental periods. Under certain conditions, parents may not feel the need, or deem it wise, to maintain a consistently high level of support for their children. One such condition occurs during the transition to young adulthood. For example, according to 
the State of California's Penal Code section 272, parents are responsible for providing "reasonable care, supervision, protection, and control over their minor child [under the age of 18]" (Graham, 2000, p. 1736). This age demarcation typically coincides with children's finishing high school and often moving away from home to attend college, work, or join the military. There is limited research on parental and peer social support around this transition. Asendorpf and Wilpers (2000) showed that the mean level of parental emotional support was similar before and after the transition to college, and Larose and Boivin (1998) reported that the mean level of peer support did not change significantly among European Americans during this transition. Nevertheless, a lack of mean differences did not signal a high level of individual stability. In fact, the stability was rather moderate (slightly higher for parental emotional support than peer emotional support in both of these studies). Instability may have resulted from parents' and peers' responses to individual adolescents' initial depressed mood. Indeed, if the lack of a causal link from early adolescents' depressed mood to parental warmth was a result of social and legal sanctions as speculated by Stice et al. (2004), one would expect that, during the transition out of high school, parents might feel less obligation to maintain their level of warmth and emotional support. Consequently, we would find that youths' depressive symptoms at this turning point in the life course would lower subsequent parental warmth, much as depression has been found to lower peer warmth.

This link between depressive mood and parental and peer warmth may vary across different ethnic and cultural groups. Several studies have examined cross-cultural differences in associations between parental and peer warmth and adolescents' depressive symptoms. These studies have included samples from countries such as the United States, China, Korea, Czech Republic (e.g., Dmitrieva, Chen, Greenberger, \& Gil-Rivas, 2004; Farruggia, Chen, Greenberger, Dmitrieva, \& Macek, 2004; Greenberger et al., 2000), Mexico (e.g., Gil-Rivas, Greenberger, Chen, \& Lopez-Lena, 2003), and other ethnic groups such as African, Asian, Hispanic, and European Americans (e.g., Eamon, 2002; Demaray, Malecki, Davidson, Hodgson, \& Rebus, 2005; Greenberger \& Chen, 1996). These mostly cross-sectional studies generally found cross-cultural similarities in the negative associations between parental and peer warmth and adolescents' depressive symptoms (see Khaleque \& Rohner, 2002, for a meta-analytic review). None of these studies, however, examined the directionality of causal relations or studied the critical transition from high school into young adulthood. Furthermore, all the longitudinal and experimental studies mentioned above that examined the possibility of erosion of social support have focused predominantly on Whites. As a result, it is not clear if or how ethnicity might have played a moderating role in their findings. It is possible that cultural differences in familistic values or beliefs about the role of parents may moderate an erosion of parental support and warmth during the transition to young adulthood. 
One can speculate that family-oriented, collectivist cultures and cultures with a later time-table for adolescent autonomy (see Feldman \& Rosenthal, 1991) would be more likely to view the role of parents as continuing after the transition than would individualistic cultures. Likewise, collectivist values might mitigate peers' withdrawal of warmth from their depressed friends. In the present study, we included three ethnic groups: European, Asian, and Hispanic Americans. The latter two groups have been found to be more familistic and collectivist than European Americans (Oyserman, Coon, \& Kemmelmeier, 2002). In light of previous studies, we hypothesized that depressive symptoms observed in high school would be negatively associated with parental and peer warmth 1 year later. We also hypothesized that the erosion of parental and peer warmth would be less evident in family-oriented, collectivist cultures (Asian and Hispanic American) than in individualistic cultures (European American).

\section{METHOD}

\section{Participants and Procedure}

Participants in the current study are part of an ongoing, larger longitudinal study of the transition out of high school. Participants were recruited at four high schools within the Los Angeles Unified School District that are characterized by a diverse working- and middle-class student population. Time 1 data $(N=1,183)$ were collected during a regular class period when the participants were high school seniors. Eighty-one percent of the enrolled students consented to participate in the study. Classroom drawings for two $\$ 20$ gift certificates and two school drawings for a $\$ 100$ gift certificate to a local record store were offered as an incentive for the students' participation. Time 2 data $(N=754)$ were collected 1 year after graduation through mailed questionnaires that participants completed and mailed back. Individuals were paid $\$ 40$ for their participation at Time 2.

The present study used a subsample of 511 students (58.7\% females) from the original sample (see Chang, Chen, Greenberger, Dooley, \& Heckhausen, 2006) who met the following requirements: They were of Asian, Hispanic, or European descent and completed key measures for this study for both waves of measurement. The ethnicities of the Asian Americans were Filipino (56.6\%), Korean (16.4\%), Vietnamese (8.6\%), Chinese (7.9\%), Japanese (3.3\%), and other Asian (7.2\%). Of the Hispanic Americans, $65.4 \%$ were Mexicans, $27.3 \%$ were Central Americans, $4.4 \%$ were South Americans, and $<1 \%$ were other Hispanics. All of the European Americans identified themselves as such and were not asked for their specific ethnicities.

Participants' mean age at Time 1 was 17.8 years $(S D=.56$, range $=17-20$ years). There were 154 European Americans (13 first generation [the adolescent was born outside of the United States]; 4 second generation [the 
adolescent was born in the United States but both parents were born outside of the United States]; and 137 third or later generation [the adolescent and at least one parent were born in the United States]). Of the 205 Hispanic Americans, 52 were first generation, 124 second generation, and 29 third or later generation. Among the 152 Asian Americans, 74 were first generation, 69 second generation, and 9 third or later generation. Not surprisingly, crossethnic differences in generational status were significant, $F(2,469)=180.74$, $p<.001$, partial $\eta^{2}=.435$. Games-Howell post hoc comparisons showed that European Americans' generational status $(M=2.83$, standard error $[S E]=.05)$ was higher than that of Hispanic Americans $(M=1.91, S E=.04)$, which in turn was higher than that of Asian Americans $(M=1.56, S E=.05)$.

The mean of parental educational attainment, averaged across mother and father, also differed across ethnic groups, $F(2,469)=128.63, p<.001$, partial $\eta^{2}=.354$. Games-Howell post hoc analyses indicated that the parents of Hispanic Americans $(M=1.96, S E=.07)$ had significantly lower educational attainment than those of both European $(M=3.54, S E=.08)$ and Asian Americans $(M=3.35, S E=.08)$.

\section{Measures}

Parental warmth. An 8-item Parental Warmth and Acceptance Scale was used to assess perceived parents' warmth, understanding, and support (Greenberger \& Chen, 1996). Each item was rated on a 6-point Likert-type scale ranging from $1=$ Strongly disagree to $6=$ Strongly agree. Sample items are "They really understand me," "I know that they will be there for me if I need them," and "They enjoy spending time with me." The convergent validity of this scale has been supported by its significant correlation with the Family Cohesion subscale of the Moos and Moos (1984) Family Environment Scale for both European Americans and Asian Americans (Greenberger \& Chen, 1996). The construct validity for Mexican adolescents was evidenced by a positive relation between this measure and a measure of parental knowledge of children's activities (Gil-Rivas et al., 2003). Scores on this scale were also negatively correlated with conflict with parents for Asian American, European American, Korean, and Chinese youths (e.g., Beam, Gil-Rivas, Greenberger, \& Chen, 2002; Greenberger \& Chen, 1996; Greenberger et al., 2000) as well as for Mexican adolescents (Gil-Rivas et al., 2003). Furthermore, measurement equivalence of this measure across cultures has been demonstrated by Chen, Farruggia, Greenberger, and Powers (2002), indicating no substantive difference in the interpretation of the scale items.

Peer warmth. Participants' perception of peer warmth, understanding, and support (Greenberger, Chen, \& Beam, 1998) was assessed using identical 
item content as in the Parental Warmth and Acceptance Scale, for example, "I know that they will be there for me if I need them." Evidence for the construct validity of this scale has been provided by Beam, Chen, and Greenberger (2002) and Greenberger et al. (2000). For example, theoretical considerations suggest that there should be a greater propensity of disclosing to friends when one perceives warmth and acceptance from them. Greenberger et al. found a positive and statistically significant correlation between this scale and a measure of problem disclosure to friends for both Chinese and American youths.

Depressive symptoms. Participants' depressive symptoms were evaluated based on their responses to the 20-item CES-D scale (Radloff, 1977). The participants reported the frequency of depressive symptoms over the past month on a 4-point Likert-type scale ranging from $1=$ Rarely or none of the time to $4=$ Most or all of the time. Sample items are "I had trouble keeping my mind on what I was doing" and "I felt that I couldn't shake off the blues even with the help of my family and friends." The psychometric properties of the CES-D have been demonstrated in studies using European Americans, Chinese, and Mexican adolescents (e.g., Crockett, Randall, Shen, Russell, \& Driscoll, 2005; Gil-Rivas et al., 2003; Greenberger et al., 2000). Furthermore, the longitudinal factorial invariance of the CES-D has been established in previous research (e.g., Motl, Dishman, Birnbaum, \& Lytle, 2005; Radloff, 1977). Evidence for measurement equivalence across European and Mexican Americans has been reported by several researchers (e.g., Crockett et al., 2005; Dmitrieva et al., 2004).

Alpha coefficients for the various scales, calculated separately for each ethnic group, ranged from .78 to .87 for the Parental Warmth and Acceptance Scale; .75 to .84 for the Peer Warmth and Acceptance Scale; and .87 to .91 for the CES-D scale.

\section{Structural Equation Modeling With Latent Variables}

Path models of the associations between parental and peer warmth and adolescents' depressive symptoms were examined using the maximum likelihood estimation method with AMOS 4.0 (Arbuckle \& Wothke, 1999). We utilized a three-construct, two-wave, crossed-lagged effect model (Burkholder \& Harlow, 2003; Kenny, 1975; Kenny \& Harackiewicz, 1979; cf. Rogosa, 1980). Figure 1 illustrates the basic model. We hypothesized that depressive symptoms, perceived parental warmth, and perceived peer warmth at Time 2 were a function of their Time 1 counterparts $\left(\beta_{11}, \beta_{22}\right.$, and $\left.\beta_{33}\right)$ and of the other constructs' influences $\left(\beta_{12}, \beta_{13}, \beta_{21}\right.$, and $\left.\beta_{31}\right)$. In addition, contemporaneous (or concurrent) variables were expected to be correlated at both time points $\left(\phi_{11}, \phi_{21}, \phi_{31}, \psi_{12}, \psi_{22}\right.$, and $\left.\psi_{32}\right)$. 


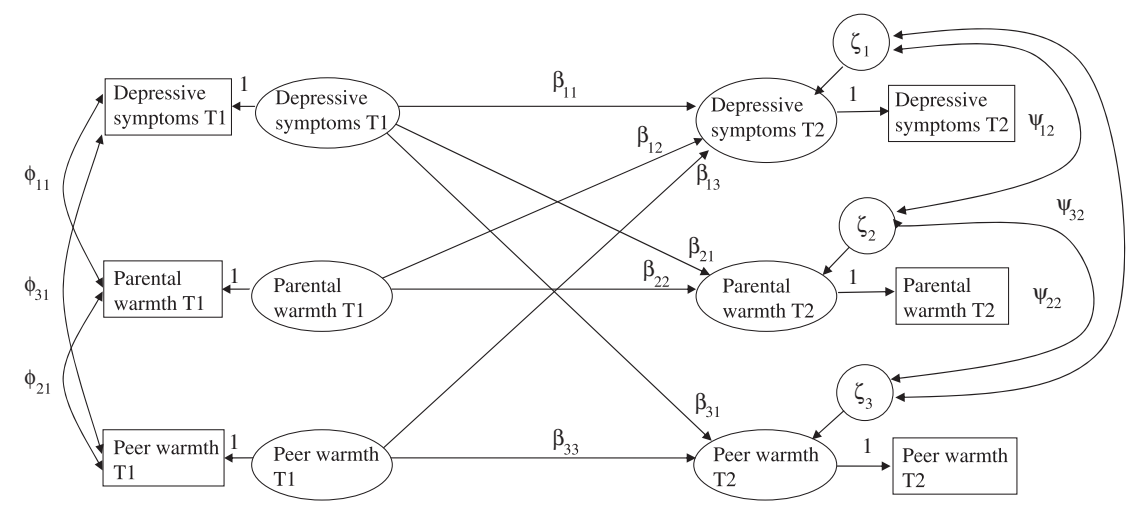

FIGURE 1 The proposed three-construct, two-wave, crossed-lagged effect model. $\mathrm{T} 1=$ Time $1 ; \mathrm{T} 2=$ Time 2 . The path between each latent variable and its sole indicator was set to 1 to scale the latent variable. The measurement error associated with its respective manifest variable was omitted for the ease of presentation.

\section{Multiple Imputation (MI)}

The rate of missing data ranged from $0 \%$ to $4 \%$ across the measures. In his simulation study of cross-lagged data, Newman (2003) showed that MI, as compared with ad hoc approaches (e.g., pairwise deletion) and the expectation-maximization (EM) algorithm, yielded the most accurate estimates of parameters and their SE. Therefore, we used the MI algorithm to handle missing data. With this algorithm, missing values were replaced by $m>1$ simulated datasets, where $m$ is usually small (e.g., 5-10). The program NORM (Schafer, 1999) was used for MI analyses in the present study. Briefly, Schafer's MI procedure involves two steps: an EM algorithm step and a data augmentation (DA) step. The EM algorithm is an iterative method with which missing values are predicted based on the available cases using regression techniques. Then the unknown parameters are calculated using the imputed data along with the non-missing data. This process repeats until the parameter estimates converge to maximum likelihood estimates. The DA step uses the maximum likelihood estimates of the parameters generated by the EM algorithm to predict missing cases and then makes a random draw of parameters from a posterior distribution.

The $m$ sets of imputed data can be analyzed using conventional statistical methods. The parameter estimates and SEs from data analyses performed on the $m$ datasets (once for each dataset) are combined to obtain a single set of results. Chi-square statistics from the analysis of the $m$ sets of imputed data can be combined using a SAS macro provided by Allison (2000). Other model fit indices (e.g., CFI and RMSEA) that cannot be combined are reported as a range across the $m$ different analyses. In the present study, we used MI to construct five complete datasets. 


\section{RESULTS}

\section{Attrition Analysis}

A $2 \times 2$ between-subject multivariate analysis of variance (ANOVA) was performed on three criterion variables: Time 1 perceived parent warmth, perceived peer warmth, and depressed mood. Predictor variables were ethnicity and a dummy variable representing participation status (attrition) at Time 2 . There was a small but statistically significant attrition effect, Wilks' $\Lambda=.983, F(3,765)=4.42, p<.05$, partial $\eta^{2}=.017$, and the attrition effect also varied somewhat by ethnicity, as shown by a significant interaction between ethnicity and attrition, Wilks' $\Lambda=.982, F(61,530)=2.33, p<.05$, partial $\eta^{2}=.009$. (Mean scores on these criterion variables also differed by ethnicity; see next section for details.) Further univariate ANOVA revealed that the attrition effect was significant for parental $F(1,767)=11.55, p<.05$, partial $\eta^{2}=.015$, and peer warmth, $F(1,767)=6.62, p<.05$, partial $\eta^{2}=.009$, but not significant for depressed mood. The interaction was significant only for parental warmth $F(2,767)=5.90, p<.05$, partial $\eta^{2}=.015$, not for peer warmth and depressed mood. Specifically, both Asian and European Americans who participated at Time 2 reported a slightly higher level of Time 1 parental warmth $(M=4.68, S E=.08$ and $M=4.93, S E=.08$, respectively) than did those who dropped out (corresponding $M=4.12, S E=.13$ and $M=4.63$, $S E=.14)$, but there was no attrition effect for Hispanic Americans $(M=4.60$, $S E=.07 ; M=4.65, S E=.08$ for the students who participated vs. dropped out, respectively). Adolescents who participated at Time 2 reported a slightly higher mean level of Time 1 peer warmth $(M=4.82, S E=.04)$ than did those who did not participate $(M=4.64, S E=.06)$.

\section{Descriptive Statistics}

We conducted a series of three-way repeated measures ANOVAs with gender and ethnic group as the between-subjects factors, and waves of data (Time 1 or Time 2) as the within-subject factor. Means and standard deviations of key variables as well as the correlations among these variables are shown in Table 1. Because there were few gender differences, results are not shown by gender. Perceived peer warmth was significantly higher at Time 2 than at Time $1, F(1,492)=28.16, p<.001$, partial $\eta^{2}=.054$. The mean levels of peer warmth were also significantly different across the three ethnic groups, $F(2,492)=5.45, p<.01$, partial $\eta^{2}=.022$. Games-Howell post hoc comparisons showed that European Americans' mean level of perceived peer warmth was significantly higher than that of Hispanic Americans across the two occasions of measurement. Furthermore, females reported a higher mean level of perceived peer warmth than did males, $F(1,492)=13.35$, $p<.001$, partial $\eta^{2}=.026$. None of the two-way or three-way interactions were significant. 
TABLE 1

Correlations Among the Key Variables and Their Means and Standard Deviations, by Ethnicity

\begin{tabular}{lllllll}
\hline Variables & 1 & 2 & 3 & 4 & 5 & Mean Item Scores (SD) \\
\hline
\end{tabular}

European Americans

1. T1-CES-D $1.88(.55)$

2. T1-parental warmth $-.35^{\text {***** }} 4.92(.94)$

3. T1-peer warmth $-.31^{\text {**** }} .34^{* * * *} \quad 4.98(.77)$

$\begin{array}{lllll}\text { 4. T2-CES-D } & .59^{* * * *} & -.25^{* * *} & -.07 & 1.82(.52)\end{array}$

5. T2-parental warmth $-.43^{* * * *} \quad .59^{* * * *} \quad .22^{* *} \quad-.43^{* * * *} \quad 4.90(.90)$

6. T2-peer warmth $\quad-.31^{* * * *} \quad .35^{* * * *} \quad .40^{* * * *}-.36^{* * * *} .57^{* * * *} \quad 5.10(.74)$

Hispanic Americans

1. T1-CES-D

$2.01(.53)$

2. T1-parental warmth $-.32^{* * * *} \quad 4.60(.91)$

3. T1-peer warmth $\quad-.36^{* * * *} \quad .45^{* * * *} \quad 4.72(.81)$

$\begin{array}{lllll}\text { 4. T2-CES-D } & .44^{* * * *} & -.27^{* * * *} & -.25^{* * * *} & 1.84(.47)\end{array}$

5. T2-parental warmth $-.16^{*} \quad .53^{* * * *} \quad .25^{* * * *}-.44^{* * * *} \quad 4.64(.87)$

6. T2-peer warmth $\quad-.23^{* * *} \quad .30^{* * * *} \quad .39^{* * * *} \quad-.39^{* * * *} \quad .39^{* * * *} \quad 4.90(.74)$

Asian Americans

1. T1-CES-D

$1.97(.50)$

2. T1-parental warmth $-.44^{* * * *} \quad 4.68(.94)$

3. T1-peer warmth $\quad-.40^{* * * *} \quad .42^{* * * *} \quad 4.76(.84)$

$\begin{array}{lllll}\text { 4. T2-CES-D } & .62^{* * * *}-.38^{* * * *}-.32^{* * * *} & 1.86(.51)\end{array}$

5. T2-parental warmth $-.47^{\text {**** }} \quad .68^{\text {**** }} \quad .32^{\text {**** }}-.40^{* * * *} \quad 4.56(.98)$

6. T2-peer warmth $\quad-.40^{* * *} \quad .26^{* * *} \quad .55^{* * * *}-.42^{* * * *} .43^{* * * *} \quad 5.02(.69)$

Notes. Nonimputed data were used to generate this table.

$\mathrm{T} 1=$ Time $1 ; \mathrm{T} 2=$ Time 2.

$* p<.05 ; * * *<.01 ; * * * * *<<.001$.

With regard to perceived parental warmth, the reported mean levels did not differ significantly between Time 1 and Time 2, $F(1,494)=.44, p=.51$, partial $\eta^{2}=.001$. The reported mean levels of parental warmth, however, differed across ethnic groups, $F(2,494)=6.58, p<.01$, partial $\eta^{2}=.026$, with European Americans reporting higher mean levels of parental warmth than did Asian and Hispanic Americans across both occasions of measurement. There were no significant gender differences in perceived parental warmth. Again, none of the two-way or three-way interactions were statistically significant.

Adolescents' depressive symptoms were lower at Time 2 than at Time 1, $F(1,503)=27.21, p<.001$, partial $\eta^{2}=.051$. The mean levels of depressive symptoms did not differ by gender or among the three ethnic groups. None of the two-way or three-way interactions were statistically significant. 


\section{Path Models}

Due to the parameter-to-sample-size ratio limitation, we used the averaged score of each scale (manifest variables) to quantify the constructs. To guard against the effect of differential measurement errors across ethnic groups, we incorporated measurement errors by following Bollen's (1989) recommendation: Each latent construct (depressive symptoms, parental warmth, and peer warmth) was represented by its respective manifest variables. The measurement error for each measured variable $\left(x_{i}\right)$ was set to (1 - reliability of $\left.x_{i}\right) \times$ variance of $x_{i}$.

The proposed path model (Figure 1) was first used to separately fit data from the three ethnic groups. Results showed an adequate fit for all three groups (see Figures 2-4 for parameter estimates and fit indices). For European Americans (Figure 2), depressive symptoms at Time 1 were negatively associated with changes in both parental and peer warmth over time. Furthermore, Time 1 peer warmth and Time 2 depressive symptoms were positively related. Taken together, the findings for European Americans suggested a bidirectional effect. In contrast, for Hispanic Americans (Figure $3)$, none of the cross-lagged paths were statistically significant. Asian Americans (Figure 4$)$ exhibited a marginal association $(p=.08)$ between depressive symptoms at Time 1 and a negative change in parental warmth, and Time 1

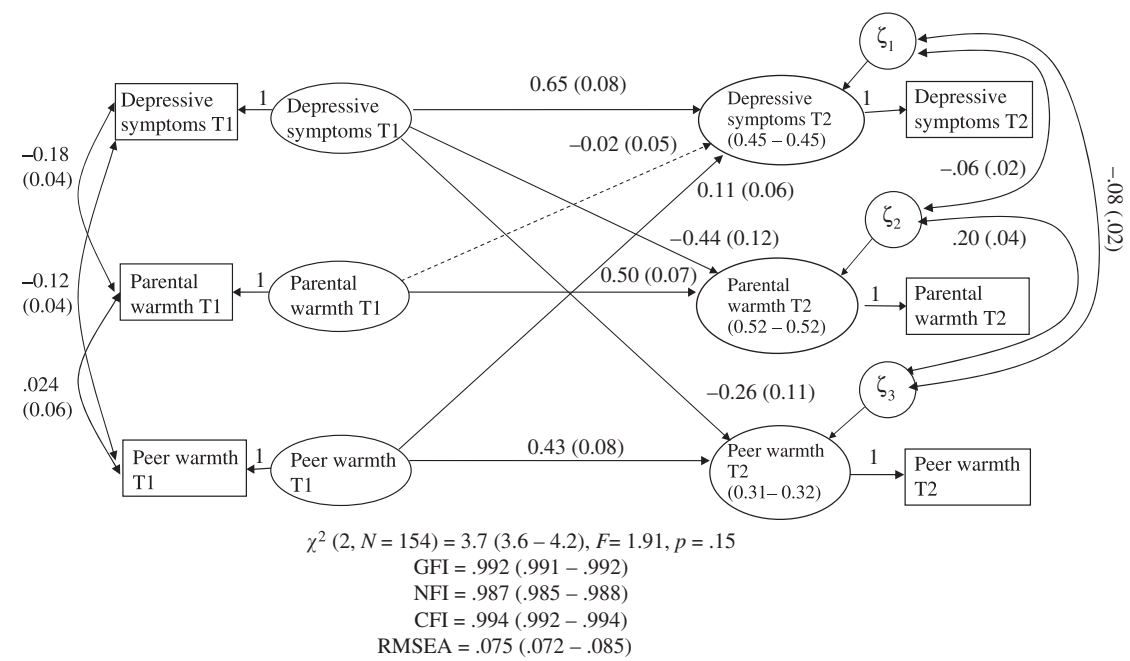

FIGURE 2 Results for the three-construct, two-wave, crossed-lagged effect model for European Americans. Solid paths depict statistically significant effects, with $p<.05$, two-tailed. Dashed paths depict nonsignificant coefficients. Unstandardized estimates with standard errors in parentheses are shown next to the pathways. $\mathrm{T} 1=$ Time $1 ; \mathrm{T} 2=$ Time 2 . Also shown are median fit indices for multiple imputation results (minimum and maximum values in parentheses). Variance explained for each of the Time 2 outcome variables is expressed in the parentheses as a range (minimum and maximum) for multiple imputation results. 


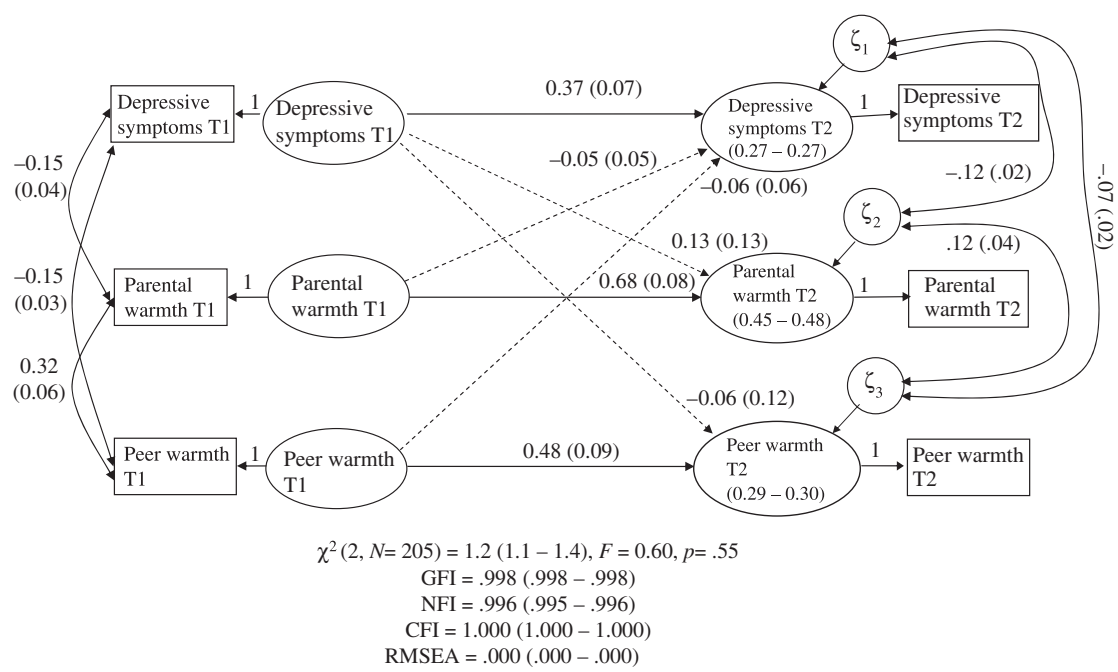

FIGURE 3 Results for the three-construct, two-wave, crossed-lagged effect model for Hispanic Americans (see Figure 2 caption for notes).

depressed mood was negatively associated with peer warmth over time. For all three groups, depressive symptoms, parental warmth, and peer warmth at Time 1 were positively associated with their counterparts at Time 2, which indicated stability of these constructs over a 1-year lag.

To test the structural equivalence of the model across the three ethnic groups, multigroup comparisons were performed. First, we performed power analysis following the steps outlined by MacCallum, Browne, and Cai

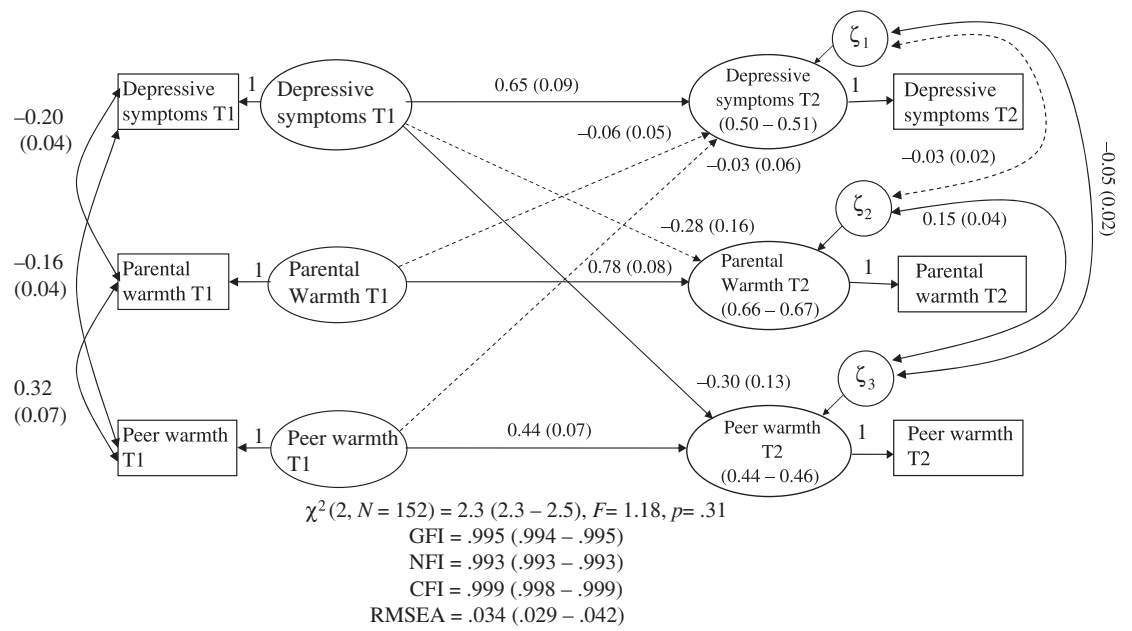

FIGURE 4 Results for the three-construct, two-wave, crossed-lagged effect model for Asian Americans (see Figure 2 caption for notes). 
(2006), with the population effect size $\delta$ specified as .04 , a commonly used number for a medium effect size (Cohen, 1988). Results showed that the power (i.e., the probability of rejecting the null hypothesis of ethnicity not being a moderator) was high (.96). Multigroup analysis for ethnicity was then conducted. Results showed that the constrained model (i.e., with all the concurrent and cross-lagged associations constrained to be equivalent across groups) had significantly worse fit to the data than did the unconstrained model. The median fit index for MI results (minimum and maximum values in parentheses) was $\Delta \chi^{2}(26)=45.2(44.2-45.9), p<.05$. After examining each path by relaxing each parameter across the three ethnic groups in the constrained model (all paths were tested for nonequivalence), we found significant ethnic differences in two paths: that between Time 1 depressive symptoms and Time 2 parental warmth, $\Delta \chi^{2}(2)=7.5(7.2-8.3), p<.05$, and that between the regression residuals associated with Time 2 depressive symptoms and Time 2 parental warmth, $\Delta \chi^{2}(2)=8.0$ (7.6-8.7), $p<.05$. Further pair-wise comparisons among ethnic groups revealed that both Asian and European Americans exhibited a stronger negative association between Time 1 depressive symptoms and Time 2 parental warmth than did Hispanic Americans, $\Delta \chi^{2}(1)=4.2(3.4-5.0), p<.05$ and $\Delta \chi^{2}(1)=10.7$ (9.9-11.1), $p<.05$, respectively. Moreover, Hispanic Americans exhibited a stronger negative partial correlation between Time 2 depressive symptoms and parental warmth than did Asian Americans, $\Delta \chi^{2}(1)=7.4$ (6.9-7.9), $p<.05$.

In sum, both European and Asian Americans (marginally for the latter) exhibited evidence for the erosion of parental warmth by initial depressed mood during the transition to young adulthood. On the other hand, Hispanic Americans' depressive symptoms at Time 1 did not have any relation to Time 2 parental warmth. In terms of peer warmth, European and Asian Americans, but not Hispanic Americans, showed a significant negative coefficient between Time 1 depressed mood and Time 2 peer warmth after controlling for Time 1 peer warmth. It should be noted that, unlike parental warmth, which did not show a change in mean levels between Time 1 and Time 2 (see "Descriptive Statistics"), the average peer warmth score actually increased somewhat over this transition (by .12 [SD =.82] on a 6-point scale for European Americans, .18 [SD = .78] for Hispanic Americans, and .26 [SD = .75] for Asian Americans; see Table 1). In other words, higher depressive symptoms at Time 1 predicted a smaller increase in peer warmth, but for those participants whose peer warmth was about .15-.35 of a standard deviation lower than the mean, "true erosion" (i.e., a decline in absolute levels of peer warmth, not merely a "smaller increase") appeared to have occurred.

\section{Potential Confounding Variables}

The present study focused on ethnic differences, but it is necessary to ascertain whether other potential moderators (e.g., gender, family SES, 
generational status) would produce major confounding effects. First, we conducted power analyses for the potential confounding moderators. Results showed that the power was high for detecting gender as a moderator (.82), but low for detecting generational status as a moderator (.26 for Asian Americans, .35 Hispanic Americans, and not relevant for European Americans due to the small number of first- and second-generation students). Therefore, multigroup comparison was conducted only for gender. The original model (Figure 1) was used to separately fit data from females and males. Results showed an adequate fit for both genders. Median fit indices for MI results (minimum and maximum values in parentheses) were $\chi^{2}(4$, $N=511)=6.4(6.0-6.9), p=.18 ; \mathrm{GFI}=.996(.996-.996) ; \mathrm{NFI}=.993(.992-.993)$; $\mathrm{CFI}=.997$ (.997-.998); RMSEA $=.034$ (.032-.037). Multigroup analysis did not show any difference between the model that constrained all the paths to be equivalent across genders and the unconstrained model, $\Delta \chi^{2}(13)=11.7$ (11.1-12.8), $p=.56$.

Although we were not able to assess the moderating effect of generational status, we could statistically control for the effects of generational status and family SES. We thus examined the relations between perceived warmth of others and depressed symptoms with family SES and generational status as covariates. We reran the analyses presented in the earlier section on path models controlling for both parental education (averaged across mother and father educational attainments) and generational status. Results based on the new models confirmed a lack of gender differences, $\Delta \chi^{2}(26)=23.1(21.8-$ 23.8), $p=.67$. The results for ethnic differences were also the same as in the original model, $\Delta \chi^{2}(52)=86.7$ (85.2-87.1), $p<.05$, except for two significant paths associated with the newly added covariates. First, there was stronger covariance between parental education and Time 1 parental warmth for Asian $(-.16 \pm .07[S E], p<.05)$ than for Hispanic Americans $(.03 \pm .14$ [SE], $\left.p=.81, \Delta \chi^{2}(1)=6.2(6.0-6.2), p<.05\right)$, and European Americans $(.15 \pm .08$ $\left.[S E], p<.05, \Delta \chi^{2}(1)=9.3(9.2-9.6), p<.05\right)$. Second, Hispanic Americans $(.13 \pm .07[S E], p=.08)$ showed stronger covariance between generational status and parental education than did European Americans $(-.08 \pm .04$ [SE], $p=.08), \Delta \chi^{2}(1)=13.2(13.2-13.2), p<.05$.

\section{DISCUSSION}

This longitudinal study investigated the temporal relations between perceived parental and peer warmth and adolescents' depressed mood during a critical point in the transition to young adulthood. Consistent with our hypothesis that erosion of both parental and peer warmth would occur for the more depressed individuals during this transition period, results showed that, for European Americans, initial depressive symptoms were negatively associated with subsequent perceived parental and peer warmth. These results replicated the findings of Stice et al. (2004) and Prinstein et al. (2005) in 
terms of the erosion of peer support and warmth and extended their findings to include parental warmth erosion during the transition to young adulthood. At this juncture, parents may feel that social proscriptions for providing emotional support to their grown children are no longer as strong, and a degree of "letting go" is acceptable, especially when their children engage them in depressive social interactions.

Also as expected, the link between depression and erosion of parental and peer warmth was not evident for one of the two cultural groups with a strong collectivist and familistic orientation-Hispanic Americans. Hispanic American parents were perceived to provide consistent emotional support and warmth regardless of the level of adolescents' depressive symptoms. Future research should address whether under certain conditions (e.g., a different transition, such as from college to work or from singlehood to marriage) or at their children's later age, even Hispanic parents adjust their support in response to their grown children's depressive symptoms.

Asian Americans-another group with a strong collectivist and familistic orientation-showed mixed evidence for our hypothesis. Depressed mood was significantly negatively associated with changes in peer warmth, but that association was only marginal for parental warmth. Several factors may account for this tentative finding. First, our results may have detected an early sign of the psychological distancing strategy used by Asian American parents to deal with their children who display depressive symptoms. Asian American families have been found to have a tendency to reject their psychologically troubled members, perhaps to protect the family reputation from the cultural stigma of having psychological problems (Uba, 1994). Future longitudinal research is needed to directly test these conjectures.

Second, our Asian American sample had a high proportion of Filipinos. According to a study on individualism and collectivism among Filipino adults, as summarized in Oyserman et al.'s (2002) meta-analysis, participants in the Philippines did not differ from Americans in their collectivist orientation.

Third, other factors, such as religiosity, may be relevant in the understanding of ethnic differences in the connections between adolescent depressive symptoms and parental and peer warmth during the transition to adulthood. ${ }^{1}$ Among Hispanics, there is strong adherence to Catholic theology that promotes the importance of, and adherence to, traditional family values. Such values emphasize solidarity and mutual assistance. Indeed, as Magaña and Clark (1995) observed, a fundamental feature of Latino religiosity "is its fascination with healing of every kind-physical, mental, and spiritual" ( $\mathrm{p}$. 103). In contrast, there is a great diversity of religions among Asian Americans (Lee, 1997), and the level of religiosity varies from very low to very high. Perhaps it is not collectivism per se, but rather familism embedded in a

\footnotetext{
${ }^{1}$ We acknowledge the contributions of anonymous reviewers for the discussion of the religiosity and residence factors.
} 
religious context that poses a strong sanction against Hispanic parents' withdrawal of warmth and support from their children when they exhibit symptoms of depression. Because there is a wide spectrum of religious beliefs within each ethnic group, researchers who wish to focus on the role of religiosity on warmth erosion should be sure to delineate this diversity.

Another factor that should be considered is adolescents' residence after the transition from high school. Residence is affected by cultural values such as familism, the family's economic situation, and distance between the parents' home and school or work, among other factors. Nevertheless, residency may offer further insights into the discussion of support erosion. Post hoc analyses of our data ${ }^{2}$ indeed revealed that more European and Asian Americans moved away from home at this transition than did Hispanic Americans, and, as noted above, that the former two ethnic groups showed evidence of parental warmth erosion.

The mean ethnic differences in parental and peer warmth we found were generally consistent with the findings of previous studies: European Americans reported higher levels of parental warmth than did their Asian and Hispanic counterparts. This pattern of results has been attributed to conflict between Asian American youth and their parents arising from "delayed autonomy" (Greenberger \& Chen, 1996). As Feldman and Rosenthal (1991) noted, individuals in collectivist societies are expected to attain independence and autonomy later than are those in individualistic societies. The generally higher level of perceived peer warmth among European Americans than Hispanic Americans may be due to the greater peer orientation in this individualistic culture (Chen, Greenberger, Lester, Dong, \& Guo, 1998).

It should be noted that our data were based exclusively on adolescents' self-reports of warmth and depressive symptoms. Thus, shared method variance or (negative) perceptual bias may have inflated the relations between depressive symptoms and warmth. It is possible that data based on other informants (e.g., parents' self-report of their warmth to the adolescents) or other methodology (e.g., researchers' naturalistic observations) might have yielded different results. The present findings need to be interpreted with this caution, and replications are needed to strengthen the validity of our findings. However, we believe that our findings provide valid information and conclusions for two reasons. First, insofar as perceptual bias was

\footnotetext{
${ }^{2}$ Results were based on the comparisons between participants' Time 1 and Time 2 responses to a question about their living arrangements (93\% of our sample provided answers to this question). More European Americans (46.0\%) and Asian Americans (42.9\%) than Hispanic Americans (12.8\%) in our sample moved away from their parents during the transition from high school to college. Although these differences in patterns of residence paralleled the greater erosion of parental warmth for European and Asian Americans, subsequent analyses with residence as a moderator did not confirm their connection [European Americans: $\Delta \chi^{2}(13)=12.4(11.8-13.4), F=0.95, p=.50$; Hispanic Americans: $\Delta \chi^{2}(13)=17.9(16.4-18.6), F=1.33, p=.19$; Asian Americans: $\Delta \chi^{2}(13)=16.6$ (16.4-17.3), $F=1.29, p=.21]$. Future research may need to use a more refined measure of residence including physical distance as well as frequency of contacts to adequately test this hypothesis.
} 
exerting its influence to the same extent at both measurement occasions, controlling for Time 1 warmth when predicting Time 2 warmth alleviated concern about shared variance to some extent. Second, previous studies (e.g., Chen et al., 1998) generally have found small differences between findings derived from single informants (e.g., adolescents' own reports on every measure) and those derived from multiple informants.

Our findings for a community sample indicated that Asian and European Americans who exhibited higher levels of depressive symptoms reported more negative changes in their parents' and peers' warmth. This process may set the stage for a vicious cycle, especially during a stressful transition, with the lowering of warmth and emotional support from parents and peers as a result of initial depressed mood leading to further elevation in depressed mood. This chain of events may have serious implications for youth's success in transitioning to college or work as well as for their long-term mental health. In any case, clinicians should be alert to possible cultural differences in the effect of depressive symptoms on the erosion of warmth from others. European Americans (and Asians to a certain extent) appear to be at greater risk for such support erosion than Hispanic Americans. A possible avenue for future research is the role of clinical levels of depression in the erosion of parental and peer warmth across time.

Future research should also consider refining the measure of peer warmth. In our measure of peer warmth, we did not consider the size, composition, and stability of the friendship network. Thus, variances associated with peer warmth could have been due to either a change in warmth from the same friends or a change of friends or both. Another direction for future research is to examine the support erosion effect as a function of the Gender $\times$ Ethnicity interaction. The ratio of sample size over the number of parameters estimated prevented us from conducting such an analysis, but a considerably larger sample would help detect reliable differences, even though previous research (e.g., Kashima et al., 1995; Rhee, Uleman, \& Lee, 1996) showed that gender differences and generational differences in collectivism, individualism, and other related constructs such as autonomy expectations were small as compared with ethnic and cultural groups.

To summarize, previous research that examined the emotional support erosion hypothesis has generated inconsistent findings, with some investigators (e.g., Slavin \& Rainer, 1990) offering evidence for erosion of family but not peer emotional support, and others (Stice et al., 2004) finding erosion of peer but not parental support. Focusing on a heretofore unexamined aspect of the transition to young adulthood, and studying both perceived parental and peer warmth, we believe that the present study provided evidence that may resolve the inconsistent findings of previous research. The findings of the present study are consistent with our theoretical claim that erosion of peer and especially parental warmth may emerge during this transition because of the social and legal re-definition of the youth. The strong 
proscription against parents' withdrawing their support and warmth from dependent children appears to weaken when youth reach the age of 18 and graduate from high school. Better models of the relations between depressed mood and the erosion of parental and peer warmth need to be contextualized in terms of a constellation of cultural factors that includes ethnicity and related variables, such as individualism/collectivism, traditional family values, and religiosity.

\section{ACKNOWLEDGMENTS}

The first and second authors contributed equally to this article. This study was supported by the Max-Planck Award for International Cooperation presented to Jutta Heckhausen, which was funded by the German Federal Ministry of Education and Research (BMBF), and by two awards from the School of Social Ecology to Jutta Heckhausen and Chuansheng Chen. We are indebted to Esther Chang, Susan Farruggia, Jared Lessard, and Laura Gil-Trejo for their valuable contributions to data collection and the day-to-day management of the overall project. We thank Scott Weaver for his statistical advice on structural equation modeling with single-indicator latent constructs. In addition, we would like to thank our many undergraduate researchers for their assistance with data collection, coding, and entry.

\section{REFERENCES}

Allison, P. D. (2000). COMBCHI. Retrieved October 11, 2005, from http://www.ssc.upenn. edu/ allison/combchi.sas

Arbuckle, J. L., \& Wothke, W. (1999). AMOS users' guide (Version 4.0) [Computer software manual]. Chicago: Small Waters Corporation.

Asendorpf, J. B., \& Wilpers, S. (2000). Attachment security and available support: Closely linked relationship quality. Journal of Social and Personal Relationships, 17, 115-138.

Beam, M. R., Chen, C., \& Greenberger, E. (2002). The nature of adolescents' relationships with their "very important" nonparental adults. American Journal of Community Psychology, 30, 305325.

Beam, M. R., Gil-Rivas, V., Greenberger, E., \& Chen, C. (2002). Adolescent problem behavior and depressed mood: Risk and protection within and across social contexts. Journal of Youth and Adolescence, 31, 343-357.

Bollen, K. A. (1989). Structural equations with latent variables. New York: John Wiley \& Sons Inc.

Brendgen, M., Wanner, B., Morin, A. J. S., \& Vitaro, F. (2005). Relations with parents and with peers, temperament, and trajectories of depressed mood during early adolescence. Journal of Abnormal Child Psychology, 33, 579-594.

Burkholder, G. J., \& Harlow, L. L. (2003). An illustration of a longitudinal cross-lagged design for larger structural equation models. Structural Equation Modeling, 10, 465-486.

Chang, E. S., Chen, C., Greenberger, E., Dooley, D., \& Heckhausen, J. (2006). The life goals of a multi-ethnic, multi-generational sample of high school seniors. Journal of Youth and Adolescence, 35, 321-332.

Chen, C., Farruggia, S. P., Greenberger, E., \& Powers, T. (2002). Measurement equivalence of the parental warmth and acceptance scale. Presented at the annual meeting of the Western Psychological Association, Irvine. 
Chen, C., Greenberger, E., Lester, J., Dong, Q., \& Guo, M.-S. (1998). A cross-cultural study of family and peer correlates of adolescent misconduct. Developmental Psychology, 34, $770-781$.

Cohen, J. (1988). Statistical power analysis for the behavioral sciences (2nd ed.). Hillsdale, NJ: Erlbaum.

Colarossi, L. G., \& Eccles, J. S. (2000). A prospective study of adolescents' peer support: Gender differences and the influence of parental relationships. Journal of Youth and Adolescence, 29, 661-678.

Compas, B. E., Wagner, B. M., Slavin, L. A., \& Vannatta, K. (1986). A prospective study of life events, social support, and psychological symptomatology during the transition from high school to college. American Journal of Community Psychology, 14, 241-257.

Coyne, J. C. (1976). Depression and the responses of others. Journal of Abnormal Psychology, 85, 186-193.

Crockett, L. J., Randall, B. A., Shen, Y.-L., Russell, S. T., \& Driscoll, A. K. (2005). Measurement equivalence of the Center for Epidemiological Studies Depression Scale for Latino and Anglo Adolescents: A national study. Journal of Consulting and Clinical Psychology, 73, 47-58.

Demaray, M. K., Malecki, C. K., Davidson, L. M., Hodgson, K. K., \& Rebus, P. J. (2005). The relationship between social support and student adjustment: A longitudinal analysis. Psychology in the Schools, 42, 691-706.

Dmitrieva, J., Chen, C., Greenberger, E., \& Gil-Rivas, V. (2004). Family relationships and adolescent psychological outcomes: Converging findings from Eastern and Western cultures. Journal of Research on Adolescence, 14, 425-447.

Eamon, M. K. (2002). Influences and mediators of the effect of poverty on young adolescent depressive symptoms. Journal of Youth and Adolescence, 31, 231-242.

Farruggia, S. P., Chen, C., Greenberger, E., Dmitrieva, J., \& Macek, P. (2004). Adolescent selfesteem in cross-cultural perspective: Testing measurement equivalence and a mediation model. Journal of Cross-Cultural Psychology, 35, 719-733.

Feldman, S. S., \& Rosenthal, D. A. (1991). Age expectations of behavioural autonomy in Hong Kong, Australian and American youth: The influence of family variables and adolescents' values. International Journal of Psychology, 26, 1-23.

Galambos, N. L., Barker, E. T., \& Krahn, H. J. (2006). Depression, self-esteem, and anger in emerging adulthood: Seven-year trajectories. Developmental Psychology, 42, 350-365.

Ge, X., Best, K. M., Conger, R. D., \& Simons, R. L. (1996). Parenting behaviors and the occurrence and co-occurrence of adolescent depressive symptoms and conduct problems. Developmental Psychology, 32, 717-731.

Gil-Rivas, V., Greenberger, E., Chen, C., \& Lopez-Lena, M. M. (2003). Understanding depressed mood in the context of a family-oriented culture. Adolescence, 38, 93-109.

Graham, P. K. (2000). Parental responsibility laws: Let the punishment fit the crime [Electronic version]. Law Review, 33, 1719-1754.

Greenberger, E., \& Chen, C. (1996). Perceived family relationships and depressed mood in early and late adolescence: A comparison of European and Asian Americans. Developmental Psychology, 32, 707-716.

Greenberger, E., Chen, C., \& Beam, M. R. (1998). The role of "very important" nonparental adults in adolescent development. Journal of Youth and Adolescence, 27, 321-343.

Greenberger, E., Chen, C., Tally, S. R., \& Dong, Q. (2000). Family, peer, and individual correlates of depressive symptomatology among U.S. and Chinese adolescents. Journal of Consulting and Clinical Psychology, 68, 209-219.

Hammen, C. L., \& Peters, S. D. (1977). Differential responses to male and female depressive reactions. Journal of Consulting and Clinical Psychology, 45, 994-1001.

Joiner, T. E. (1999). A test of interpersonal theory of depression in youth psychiatric inpatients. Journal of Abnormal Child Psychology, 27, 77-85. 
Kashima, Y., Yamaguchi, S., Kim, U., Choi, S.-C., Gelfand, M. J., \& Yuki, M. (1995). Culture, gender, and self: A perspective from individualism-collectivism research. Journal of Personality and Social Psychology, 69, 925-937.

Kenny, D. A. (1975). Cross-lagged panel correlation: A test for spuriousness. Psychological Bulletin, 82, 887-903.

Kenny, D. A., \& Harackiewicz, J. M. (1979). Cross-lagged panel correlation: Practice and promise. Journal of Applied Psychology, 64, 372-379.

Khaleque, A., \& Rohner, R. P. (2002). Perceived parental acceptance-rejection and psychological adjustment: A meta-analysis of cross-cultural and intracultural studies. Journal of Marriage and Family, 64, 54-64.

King, D. A., \& Heller, K. (1984). Depression and the response of others: A re-evaluation. Journal of Abnormal Psychology, 93, 477-480.

Kubik, M. Y., Lytle, L. A., Birnbaum, A. S., Murray, D. M., \& Perry, C. L. (2003). Prevalence and correlates of depressive symptoms in young adolescents. American Journal of Health Behavior, 27, 546-553.

Larose, S., \& Boivin, M. (1998). Attachment to parents, social support expectations, and socioemotional adjustment during the high school-college transition. Journal of Research on Adolescence, 8, 1-27.

Lee, E. (Ed.). (1997). Overview: The assessment and treatment of Asian American families. In E. Lee (Ed.), Working with Asian Americans: A guide for clinicians (pp. 3-36). New York: Guilford Press.

MacCallum, R. C., Browne, M. W., \& Cai, L. (2006). Testing differences between nested covariance structure model: Power analysis and null hypotheses. Psychological Methods, 11, 19-35.

Magaña, A., \& Clark, N. M. (1995). Examining a paradox: Does religiosity contribute to positive birth outcomes in Mexican American populations? Health Education Quarterly, 22, 96-109.

Moos, R. H., \& Moos, B. S. (1984). Family environment scale manual (Rev. ed.). Palo Alto, CA: Consulting Psychologists Press.

Motl, R. W., Dishman, R. K., Birnbaum, A. S., \& Lytle, L. A. (2005). Longitudinal invariance of the Center for Epidemiologic Studies-Depression Scale among boys and girls in middle school. Educational and Psychological Measurement, 65, 90-108.

Newman, D. A. (2003). Longitudinal modeling with randomly and systematically missing data: A simulation of ad hoc, maximum likelihood, and multiple imputation techniques. Organization Research Methods, 6, 328-362.

Oyserman, D., Coon, H. M., \& Kemmelmeier, M. (2002). Rethinking individualism and collectivism: Evaluation of theoretical assumptions and meta-analysis. Psychological Bulletin, 128, 3-72.

Parker, J. G., \& Asher, S. R. (1993). Friendship and friendship quality in middle childhood: Links with peer group acceptance and feelings of loneliness and social dissatisfaction. Developmental Psychology, 29, 611-621.

Prinstein, M. J., Borelli, J. L., Cheah, C. S. L., Simon, V. A., \& Aikins, J. W. (2005). Adolescent girls' interpersonal vulnerabilty to depressive symptoms: A longitudinal examination of reassurance-seeking and peer relationships. Journal of Abnormal Psychology, 114, 676-688.

Radloff, L. S. (1977). The CES-D scale: A self-report depression scale for research in the general population. Applied Psychological Measurement, 1, 385-401.

Rhee, E., Uleman, J. S., \& Lee, H. K. (1996). Variations in collectivism and individualism by ingroup and culture: Confirmatory factor analyses. Journal of Personality and Social Psychology, 71, 1037-1054.

Rogosa, D. (1980). A critique of cross-lagged correlation. Psychological Bulletin, 88, 245-258.

Rohner, R. P. (2004). The parental "acceptance-rejection syndrome": Universal correlates of perceived rejection. American Psychologist, 59, 830-840.

Schafer, J. L. (1999) NORM: Multiple imputation of incomplete multivariate data under a normal model, version 2. Software for Windows 95/98/NT. Retrieved July 11, 2005, from http:// www.stat.psu.edu/_jls/misoftwa.html 
Sheeber, L., Hops, H., Alpert, A., Davis, B., \& Andrews, J. (1997). Family support and conflict: Prospective relations to adolescent depression. Journal of Abnormal Child Psychology, 25, 333344.

Slavin, L. A., \& Rainer, K. L. (1990). Gender differences in emotional support and depressive symptoms among adolescents: A prospective analysis. American Journal of Community Psychology, 18, 407-421.

Stice, E., Ragan, J., \& Randall, P. (2004). Prospective relations between social support and depression: Differential direction of effects for parental and peer support? Journal of Abnormal Psychology, 113, 155-159.

Uba, L. (1994). Asian Americans: Personality patterns, identity, and mental health. New York: The Guilford Press.

Young, J. F., Berenson, K., Cohen, P., \& Garcia, J. (2005). The role of parent and peer support in predicting adolescent depression: A longitudinal community study. Journal of Research on Adolescence, 15, 407-423. 